\title{
Co-aggregation of ovalbumin and lysozyme
}

Kazuki Iwashita ${ }^{a}$, Akihiro Handa $^{b}$, Kentaro Shiraki $^{a *}$

${ }^{a}$ Faculty of Pure and Applied Sciences, University of Tsukuba, 1-1-1 Tennodai, Tsukuba, Ibaraki 305-8573, Japan

${ }^{b}$ Institute of Technology, R\&D Division, Kewpie Corporation, 2-5-7 Sengawa, Chofu, Tokyo 182-0002, Japan

* To whom correspondence should be addressed.

Telephone: +81-29-853-5306;

Fax: +81-29-853-5215;

E-mail: shiraki@bk.tsukuba.ac.jp 


\begin{abstract}
Hen egg white has excellent heat-induced gelation properties. However, the molecular mechanisms underlying the aggregation of egg white proteins have not been elucidated due to their complex composition. Here, we focused on the thermal co-aggregation process of the main component, ovalbumin (OVA), with well-studied lysozyme (LYZ) in terms of protein composition, aggregation rate, intermolecular forces, and morphology. Size exclusion chromatographic analysis of OVA-LYZ mixture by heat treatment at $70^{\circ} \mathrm{C}$ indicated that the aggregation-rate constant of LYZ increased 64-fold in the presence of equimolar OVA. In contrast, the aggregation rate of OVA was not dependent on the presence of LYZ. Enzyme assay and SDS-PAGE analysis showed that LYZ forms precipitates with unfolded OVA via reversible non-covalent interactions and irreversible disulfide bonds. The unfolding of OVA triggers co-aggregation by exposure of the aggregation-prone region, followed by disulfide bond exchange between OVA and LYZ. LYZ links covalently to small OVA aggregates through disulfide bonds, leading to the hierarchical growth of OVA-LYZ aggregates with larger networks. These results provide information regarding the thermal co-aggregation of proteins in hen egg white.
\end{abstract}

Keywords: ovalbumin; lysozyme; thermal aggregation; unfolding; specific affinity; sulfhydryl-disulfide exchange 


\section{Introduction}

Hen egg white is one of the most prominent protein source foods used as an ingredient by many food industries because of its functional properties: emulsification, foaming, and gelation. Heat coagulation is an especially important functional property of egg white protein (Mine, 1995). The physical attributes of a heat-induced gel are highly dependent on the microstructure (Handa, Takahashi, Kuroda, \& Froning, 1998). The network in the microstructure determines the appearance and texture of egg white gels by entrapping water (Nieuwland, Bouwman, Pouvreau, Martin, \& de Jongh, 2016; Urbonaite, de Jongh, van der Linden, \& Pouvreau, 2015) and modulating viscoelasticity (Tomczyńska-Mleko, Nishinari, \& Handa, 2014).

The protein network is mainly mediated by crosslinkage of disulfide bonds, and non-covalent hydrophobic and electrostatic interactions (L. Campbell, Raikos, \& Euston, 2003). The disulfide bonds and sulfhydryl groups play a crucial role in covalent crosslinking and stabilizing the gel structure of proteins (Van der Plancken, Van Loey, \& Hendrickx, 2005). In addition, the hydrophobic and electrostatic interactions control the physical characteristics of protein gels (Sun \& Hayakawa, 2002). These intermolecular forces are attributed to solution $\mathrm{pH}$, ionic strength, and co-solvents leading to promotion of protein unfolding, enhancement of hydrophobic interactions, decrease in number of ionic bonds, and destruction of disulfide bonds (Handa et al., 1998; Mine, Noutomi, \& Haga, 1990). The mechanisms underlying thermal gelation and aggregation of egg white proteins have been investigated using isolated components, such as ovalbumin (Croguennec, Renault, Beaufils, Dubois, \& Pezennec, 2007; Nieuwland et al., 2016; Tani et al., 1997; Weijers, Barneveld, Cohen Stuart, \& Visschers, 2003), ovotransferrin (Mizutani, Chen, Yamashita, Hirose, \& Aibara, 2006; Yamashita, Ishibashi, Hong, \& Hirose, 1998), lysozyme (Matsuoka, Hamada, Matsumoto, \& Shiraki, 2009; Matsuoka, Tomita, Hamada, \& Shiraki, 2007; Shiraki, Kudou, Fujiwara, Imanaka, \& Takagi, 2002), and ovomucin (Offengenden \& Wu, 2013). However, analysis of a single protein alone isolated from 
egg white is too simple to gain an understanding of the molecular mechanisms occurring in egg white aggregation. On the other hand, whole egg white is too complex to understand the aggregation process of respective proteins (Handa, Hayashi, Shidara, \& Kuroda, 2001; Iwashita, Inoue, Handa, \& Shiraki, 2015) because it contains various kinds of proteins with various molecular weights, isoelectric points, and concentrations (Guérin-Dubiard et al., 2006). To understand the structural changes occurring in egg white by heat treatment, it is necessary to elucidate the intermolecular interactions between heterogeneous proteins.

Ovalbumin (OVA) is a major protein comprising $54 \%$ of total egg white proteins. OVA is a globular protein with molecular mass of $45.5 \mathrm{kDa}$ containing one disulfide bond, four sulfhydryl groups, and zero to two phosphoryl groups with a carbohydrate chain (Huntington \& Stein, 2001). OVA is a dominant factor in the heat-induced gel formation of egg white that forms amorphous or linear aggregates (Kawachi, Kameyama, Handa, Takahashi, \& Tanaka, 2013; Yamashita et al., 1998). Lysozyme (LYZ) is one of the most abundant basic proteins in egg white with molecular mass of $14.3 \mathrm{kDa}$ comprising $3.5 \%$ of total egg white proteins; Another basic protein is avidin of $0.05 \%$. The tertiary structure of LYZ is stabilized by four disulfide bonds (De Bernardez Clark, Hevehan, Szela, \& Maachupalli-Reddy, 1998). LYZ has a melting temperature of around $80^{\circ} \mathrm{C}$ at neutral $\mathrm{pH}$ and forms aggregates irreversibly with a single exponential process during heat treatment (Kudou, Shiraki, Fujiwara, Imanaka, \& Takagi, 2003). Thus, the thermal aggregation of LYZ has been used as a model system to develop suppressors of protein aggregation, such as amino acid derivatives (Shiraki et al., 2004; Shiraki, Kudou, Sakamoto, Yanagihara, \& Takagi, 2005), amine compounds (Okanojo, Shiraki, Kudou, Nishikori, \& Takagi, 2005), and ammonium ions (Hirano et al., 2007). LYZ is a basic protein, and therefore it tends to associate electrostatically with other acidic proteins, such as $\alpha$-lactalbumin (Nigen, Croguennec, \& Bouhallab, 2009), ovomucin (Matsuda, Watanabe, \& Sato, 1982), ovotransferrin (Lechevalier et al., 2003), and OVA (Le Floch-Fouéré et al., 2009; Yu, Yao, Jiang, \& Zhang, 2006). 
Here, we investigated the co-aggregation reaction steps of an OVA-LYZ binary system. Egg white is composed of many complex constituents, containing hundreds of proteins (Stevens, 1991; Mann, 2007). Thus, heat-induced gel formation of egg white is quite difficult to understand, although the boiled egg is a familiar food. OVA and LYZ are known to spontaneously associate with each other at extremely low ionic strength even at room temperature (Le Floch-Fouéré et al., 2009; Yu, et al. 2006). In response to this fact, we selected two important proteins, OVA that is the main component of egg white and LYZ that is the most studied protein in the aggregation process. Bouhallab and Croguennec (2014) reported an overview of the induced aggregation and spontaneous reversible assembly of food proteins. By contrast, we investigated irreversible thermal aggregation of OVA-LYZ binary system by focused dynamics in the aggregation process. The co-aggregation of OVA and LYZ was reported 30 years ago (Matsudomi, Yamamura, \& Kobayashi, 1986, 1987), and the results showed that the heat-induced aggregation between OVA and LYZ is due to electrostatic interaction and disulfide bond exchange. However, the dynamics in the co-aggregation processes is still not revealed in detail. Inspired by the pioneering work of Matsudomi and co-workers, we analyzed the quantitative compositions of proteins to explore the role played by each protein during the hierarchical processes of thermal aggregation using the combination of current techniques. The interactions of proteins during heat treatment should let us control network formation of heat-induced egg white gels and aggregates. The aggregation process of OVA and LYZ was identified in terms of aggregation rates, aggregation forces, and aggregate morphology.

\section{Materials and methods}

\subsection{Materials}

Hen egg white ovalbumin (grade V) and lysozyme (six times crystallized and 
lyophilized) were obtained from Sigma Chemical Co. (St. Louis, MO). The proteins were used without further purification. Na-phosphate, Na-hydroxide, and Micrococcus luteus were obtained from Wako Pure Chemical Inc. Ltd. (Osaka, Japan).

\subsection{Sample preparation for thermal aggregation of ovalbumin and lysozyme mixtures}

Sample preparation for investigation of the aggregation rate depending on the co-existing opposite protein concentration was as follows. A solution of $50 \mu \mathrm{M}$ ovalbumin (OVA) and lysozyme (LYZ) with $0-50 \mu \mathrm{M}$ LYZ and OVA, respectively, in $50 \mathrm{mM}$ Na-phosphate buffer $(\mathrm{pH} 7.0)$ was heated at $70^{\circ} \mathrm{C}$ for various periods. The samples were centrifuged at $15000 \times \mathrm{g}$ for $20 \mathrm{~min}$, and then the soluble protein concentration in the supernatant was determined by size exclusion chromatography.

The sample preparation for investigation of the affinity of OVA and LYZ was performed as follows. A solution of $100 \mu \mathrm{M}$ OVA and LYZ containing $50 \mathrm{mM}$ Na-phosphate buffer ( $\mathrm{pH}$ 7.0) was heated at $70^{\circ} \mathrm{C}$ for $30 \mathrm{~min}$. The sample solution was diluted 2 -fold with buffer solution or mixed with another protein solution at a ratio of 1:1 at room temperature. Immediately, the sample was centrifuged at $15000 \times \mathrm{g}$ for $20 \mathrm{~min}$, and then the soluble protein concentration in the supernatant was determined by size exclusion chromatography. The sample solution before centrifugation was analyzed by electrophoresis and enzyme assay.

The sample preparation for investigation of the protein composition and morphology of aggregates was performed as follows. A mixture of OVA and LYZ with total protein concentration of $100 \mu \mathrm{M}$ in the molar fraction of $0-1$ in $50 \mathrm{mM}$ Na-phosphate buffer (pH 7.0) was heated at $70^{\circ} \mathrm{C}$ for $30 \mathrm{~min}$. The samples were centrifuged at $15000 \times \mathrm{g}$ for $20 \mathrm{~min}$ for determination of protein concentration by size exclusion chromatography. The sample solution before centrifugation was diluted 10 -fold with pure water for imaging by electron microscopy.

\subsection{Determination of soluble protein concentration using size exclusion chromatography}


Soluble protein concentration was determined by high-performance liquid chromatography (HPLC) (Shimadzu, Kyoto, Japan) using a system comprised of a degasser (DGU-20A $\mathrm{A}_{3}$, a pump (LC-10AT), an auto injector (SIL-10AxL), a column oven (CTO-10A), a UV-vis detector (SPD-10AV), and a system controller (SCL-10Avp) with a size exclusion column ( $3 \mu \mathrm{m}, 300 \mathrm{~mm} \times 7.8 \mathrm{~mm}$ i.d., Yarra SEC 3000; Phenomenex, Torrance, CA). Isocratic HPLC was conducted with a flow rate of $1.0 \mathrm{~mL} \mathrm{~min}^{-1}$ at $30^{\circ} \mathrm{C}$ using $150 \mathrm{mM}$ Na-phosphate buffer (pH 7.0). Aliquots of $30 \mu \mathrm{L}$ of samples were loaded into the column. The absorbance was monitored at $280 \mathrm{~nm}$. All soluble protein concentrations were determined as the averages of three experiments.

\subsection{Sodium dodecyl sulfate-polyacrylamide gel electrophoresis}

The protein solutions were subjected to heat treatment and mixing, and were then mixed with $125 \mathrm{mM}$ Tris-HCl (pH 6.8) loading buffer solution containing 4\% (w/v) sodium dodecyl sulfate (SDS), $10 \%(\mathrm{w} / \mathrm{v})$ sucrose, and $0.01 \%(\mathrm{w} / \mathrm{v})$ bromophenol blue with or without $10 \%(\mathrm{v} / \mathrm{v}) \beta$-mercaptoethanol at a ratio of $1: 1$. The samples were incubated for 20 hours at $25^{\circ} \mathrm{C}$ and then subjected to SDS-polyacrylamide gel electrophoresis (SDS-PAGE) using a 5\%-20\% gradient gel (e-PAGEL; ATTO Co., Tokyo, Japan) with a molecular weight marker (Precision Plus Protein Dual Xtra Standards; Bio-Rad, Hercules, CA). The gels were then stained using Coomassie Brilliant Blue R-250.

\subsection{Lysozyme enzyme assay}

Lysozyme activities catalyze the cleavage of the $\beta$-1,4-glycosidic linkages between $\mathrm{N}$-acetylmuramic acid and N-acetyl-D-glucosamine, which are in peptidoglycan cell wall of bacteria. A substrate solution of $1990 \mu \mathrm{L}$ containing $0.3 \mathrm{mg} / \mathrm{mL}$ M. luteus in $50 \mathrm{mM}$ Na-phosphate buffer ( $\mathrm{pH}$ 7.0) was mixed with $10 \mu \mathrm{L}$ of protein solution. The enzymatic reaction rate was estimated from the slope of the initial decrease in the absorbance at $600 \mathrm{~nm}$ using a 
spectrophotometer (V-630; Japan Spectroscopic Co. Ltd., Tokyo, Japan)

\subsection{Imaging of aggregates by transmission electron microscopy}

Aliquots of $4 \mu \mathrm{L}$ of protein solution were negatively stained with $4 \mu \mathrm{L}$ of $1 \%(w / v)$ tungstosilicic acid solution. Then, $4 \mu \mathrm{L}$ of the stained solution was placed on a 150 -mesh copper grid covered with a carbon-coated hydrophilic film. The solution on the grid was dried for a few minutes. The samples were observed using a transmission microscope (H7650; Hitachi, Tokyo, Japan) with an acceleration voltage of $80 \mathrm{keV}$.

\section{Results}

\subsection{Condition of $O V A$ and $L Y Z$}

Before the main experiment, we confirmed the solution structure of OVA dissolved in $50 \mathrm{mM}$ Na-phosphate buffer solution at $\mathrm{pH} 7.0$ where is the $\mathrm{pH}$ of fresh egg white. Size exclusion chromatography showed that the OVA solution contained mainly the monomeric form with small amounts of dimers, trimers, and tetramers at the elution point around $10 \mathrm{~min}$ (Supplementary Information, Fig. S1). In addition, soluble aggregates were observed at an elution time of around $5 \mathrm{~min}$. The amount of soluble aggregates was about $14 \%$, as calculated from the chromatogram peak area. The polydispersed OVA molecules were observed as expected in the crude state of egg white proteins, as described previously (Campanella, Onor, D'Ulivo, Giannarelli, \& Bramanti, 2014). According to the data, it is estimated that $50 \mu \mathrm{M}$ OVA solution contains $43.2 \pm 1.0 \%$ soluble molecules in monomeric to tetrameric forms.

Protein unfolding was monitored by the ellipticity at $222 \mathrm{~nm}$ that indicates a content of $\alpha$-helix structure. Far-UV CD analysis showed that the secondary structures of OVA and LYZ were slightly perturbed at $70^{\circ} \mathrm{C}$ (Supplementary Information, Fig. S2). Accordingly, a small amount of heat-induced unfolded molecules was thought to be present during heat treatment at 
$70^{\circ} \mathrm{C}$. Heat treatment at $70^{\circ} \mathrm{C}$ was adopted in this investigation because aggregation reaction is not too fast to analyze co-aggregation of OVA and LYZ.

\subsection{Co-aggregation of OVA and LYZ}

To understand the co-aggregation of OVA and LYZ, we investigated the heat-induced aggregation of protein mixtures measured by size exclusion chromatography. Briefly, solutions containing $50 \mu \mathrm{M}$ OVA with $0-50 \mu \mathrm{M} \mathrm{LYZ}$, and vice versa, were heated at $70^{\circ} \mathrm{C}$ for various periods. The concentrations of soluble protein are plotted in Fig. 1. The soluble OVA concentration decreased from $100 \%$ to $50 \%$ during heat treatment for $30 \mathrm{~min}$ in the presence or absence of LYZ at any concentration (Fig. 1A). As shown in the figure, the aggregation of OVA was not affected by the presence of LYZ. In contrast, the soluble concentration of LYZ depended on the concentration of OVA (Fig. 1B). In the absence of OVA, almost all of the LYZ remained in the soluble fraction after heat treatment for $30 \mathrm{~min}$. In contrast, the aggregation rate of LYZ increased with increasing concentration of mixed OVA (Fig. 1B). For example, $50 \mu \mathrm{M}$ LYZ added over $30 \mu \mathrm{M}$ OVA showed complete aggregation with heat treatment for $30 \mathrm{~min}$. For quantitative comparison, the rate constants of aggregation were determined by fitting to the exponential function. Figure 2 shows the rate constant of the aggregation processes of OVA mixed with LYZ, and vice versa. The aggregation-rate constants of OVA remained constant at about $0.12 \mathrm{~min}^{-1}$ regardless of the co-existence of LYZ (Fig. 2A). In contrast, the aggregation-rate constants of LYZ increased monotonically from $0.01 \mathrm{~min}^{-1}$ to $0.64 \mathrm{~min}^{-1}$ with increasing amount of co-existing OVA (Fig. 2B). The aggregation rate constant of LYZ with equimolar OVA was 64-fold higher than that of LYZ alone.

\subsection{Affinity between $O V A$ and $L Y Z$}

To understand the structures of LYZ and OVA during co-aggregation, we prepared proteins in the native state and heated state, and analyzed the affinity between native OVA 
(nOVA), native LYZ (nLYZ), heated OVA (hOVA), and heated LYZ (hLYZ) by evaluating instant association of OVA and LYZ. Briefly, solutions of $100 \mu \mathrm{M}$ OVA and LYZ in $50 \mathrm{mM}$ Na-phosphate buffer ( $\mathrm{pH} 7.0$ ) were individually heated at $70^{\circ} \mathrm{C}$ for $30 \mathrm{~min}$. Subsequently, the native or heated OVA and LYZ solutions were mixed with each other at a ratio of 1:1 at room temperature, and then soluble proteins in the mixed samples and control samples were analyzed by size exclusion chromatography. In addition, nOVA and nLYZ were mixed and then heated at $70^{\circ} \mathrm{C}$ for $30 \mathrm{~min}$, which was designated as "co-hOVA-LYZ." The samples were centrifuged at $15000 \times g$ for $20 \mathrm{~min}$, and then the soluble fraction was subjected to size exclusion chromatography.

Figure 3 shows the size exclusion chromatograms of the various combinations of native and heated OVA and LYZ. First, we compared the single samples of OVA and LYZ. The chromatogram peaks of nOVA appeared at around $5 \mathrm{~min}, 9 \mathrm{~min}$, and $10 \mathrm{~min}$, which corresponded to soluble aggregates, oligomer, and monomer, respectively. The chromatogram peak of $\mathrm{nLYZ}$ was observed only at $12 \mathrm{~min}$ as a monomeric form of LYZ. The amount of soluble aggregates of hOVA was increased compared to that of nOVA. The peak area at $12 \mathrm{~min}$ of hLYZ was decreased compared to nLYZ, but no other peaks were observed corresponding to soluble aggregates.

Next, we compare the mixed samples. The sample "nOVA + nLYZ" showed simple peaks composed of the peaks of nOVA and nLYZ. Similar data were obtained for "nOVA + hLYZ"; i.e., the peaks of this sample were identical to the addition of the peaks of nOVA and hLYZ. These results indicate that native OVA did not interact with native LYZ or heated LYZ. In contrast, the "hOVA + nLYZ" and "hOVA + hLYZ" samples showed two peaks of monomeric OVA and LYZ, and the soluble aggregate peak vanished. These results suggest that the soluble aggregates of OVA formed insoluble precipitates with LYZ molecules. Finally, the chromatograms of "co-hOVA-LYZ" showed only a single peak of monomeric OVA.

For quantitative comparison, we calculated the protein concentrations from the 
chromatograms (Fig. 4). After heat treatment, the soluble concentrations of OVA and LYZ decreased to $53 \%$ and $84 \%$, respectively. The soluble protein concentrations of OVA and LYZ in "nOVA + nLYZ" and "nOVA + hLYZ" samples remained constant. In contrast, the soluble protein concentrations in "hOVA + nLYZ" and "hOVA + hLYZ" samples were clearly decreased; the concentrations of both proteins were about $50 \%$. The identical concentrations of soluble LYZ and OVA indicated that the precipitates were composed of equimolar amounts of LYZ and OVA. This result indicated that both $\mathrm{nLYZ}$ and hLYZ have specific affinity for the unfolded OVA rather than native OVA. Note that the specific affinity of LYZ toward hOVA was not observed for bovine serum albumin (BSA) (Supplementary Information, Fig. S3 and S4). However, as shown in the "co-hOVA-LYZ" sample, LYZ aggregated completely on co-heating with OVA, forming aggregates at a ratio of 1:2 of OVA and LYZ. These results indicate that the specific affinity of LYZ for the heat-induced unfolded OVA could not be fully described by OVA-LYZ co-aggregation. The mechanisms were investigated as follows.

\subsection{Non-covalent and covalent bonding between LYZ and unfolded OVA}

Disulfide bond exchange is one of the most important reactions in the thermal aggregation of proteins (Tomita \& Shiraki, 2011; Shiraki, Tomita, \& Inoue, 2016). To investigate the disulfide bond exchange reaction, we performed SDS-PAGE analyses of reduced or non-reduced samples of OVA-LYZ mixture using $\beta$-mercaptoethanol (Fig. 5). Clear bands were obtained with monomeric OVA $(45 \mathrm{kDa})$ and monomeric LYZ $(14 \mathrm{kDa})$. Other minor bands were observed with dimeric $(90 \mathrm{kDa})$ and trimeric $(135 \mathrm{kDa})$ OVA. For the samples except for "co-hOVA-LYZ," all bands in mixtures of OVA and LYZ under reducing and non-reducing conditions were identical to the control samples. The broad band observed in hOVA-containing sample under non-reducing condition seems to be corresponding to denatured multimeric OVA with disulfide crosslinkage, containing in a soluble aggregate fraction from HPLC (Fig. 3). The data indicated that disulfide bond exchange between OVA and LYZ does 
not occur under conditions of ambient temperature, and that binding between hOVA and nLYZ or hLYZ is stabilized by non-covalent interactions. In contrast, the bands of the "co-hOVA-LYZ" sample under non-reducing conditions were different from those under reducing conditions; the monomeric LYZ band and a broad band of over $100 \mathrm{kDa}$ corresponding to soluble large aggregates disappeared. Note that similar intermolecular disulfide bonds were also observed between BSA and LYZ during heat treatment (Supplementary Information, Fig. S5). Therefore, intermolecular disulfide bond exchange is one of the key interactions to form insoluble aggregates of "co-hOVA-LYZ" samples during heat treatment.

\subsection{Enzyme activity of LYZ associated with OVA}

The chromatograms and SDS-PAGE analyses showed that the native state of LYZ has the ability to associate with the heat-induced unfolded state of OVA. To confirm the tertiary structure of LYZ associated with OVA, the enzyme reaction of LYZ mixed with OVA was measured. Figure 6 shows the enzyme reaction rate of LYZ. As expected, the enzyme-reaction rate of "nOVA + nLYZ" did not change compared with that of LYZ alone. Surprisingly, the enzyme reaction rate of "hOVA + nLYZ" remained at $92 \%$, while the soluble concentration of LYZ decreased to $52 \%$ (Fig. 4). Note that the enzyme reaction rate was measured by using the mixture solution containing the insoluble fraction, while the soluble protein concentration was determined from the supernatant of the mixture after centrifugation. Under conditions similar to those used for determination of soluble protein concentration, the enzyme-reaction rate of LYZ in the soluble fraction of "hOVA + nLYZ" obtained by a centrifugation was $50 \%$ corresponding to soluble protein concentration. Thus, the native LYZ appeared to contain insoluble aggregates in the "hOVA + nLYZ" sample.

\subsection{Composition and morphology of co-heated OVA-LYZ aggregates}

Subsequently, we investigated the protein compositions of OVA and LYZ for 
collaborative aggregation. Briefly, mixtures of OVA and LYZ with total protein concentration of $100 \mu \mathrm{M}$ in molar fractions of $0.0-1.0$ were heated at $70^{\circ} \mathrm{C}$ for $30 \mathrm{~min}$, and the residual soluble protein concentration was then determined by size exclusion chromatography (Fig. 7A). The soluble OVA concentration after heating was directly proportional to the initial OVA concentration. In contrast, the soluble LYZ concentration decreased markedly with increasing molar fraction of OVA. At OVA molar fraction $>0.3$, LYZ was completely aggregated. To present the data more clearly, the protein compositions of OVA and LYZ in aggregates are plotted (Fig. 7B). The composition of LYZ was abundant in aggregates under all conditions. In particular, when the overall molar fraction of OVA was 0.5, the protein composition in aggregates showed 30\% OVA. Therefore, LYZ heated with OVA was prone to form aggregates due to intermolecular disulfide bonds, resulting in high proportions of LYZ in aggregates.

The morphologies of the aggregates formed in OVA-LYZ mixtures at various molar ratios were examined by TEM (Fig. 8). The aggregates of LYZ alone $(X=0.0)$ were small and amorphous, ranging in size from $10 \mathrm{~nm}$ to $100 \mathrm{~nm}$ (Fig. 8A). At $X=0.1$, large aggregates were observed in TEM images with a size range from $10 \mathrm{~nm}$ to $1 \mu \mathrm{m}$ (Fig. 8B). With increasing molar fraction of OVA at $X=0.3$, the small aggregates were not observed, but large aggregates of few $\mu \mathrm{m}$ in size were found in TEM images (Fig. 8C). In the solution containing equimolar amounts of OVA and LYZ at $X=0.5$, the aggregates apparently grew to above several dozens $\mu \mathrm{m}$ with a large network (Fig. 8D). With further increases in the ratio of OVA, the smaller aggregates increased with a spherical shape (Fig. 8 E-G). The aggregate size formed at $X=0.7-0.9$, which is similar ratio to the actual egg white protein composition, was a little larger than at $X=1.0$. Judging from the morphology, the aggregation mode of OVA-LYZ mixtures was probably dominated by (i) LYZ aggregate-driven amorphous shapes when $X<0.5$ or (ii) OVA aggregate-driven spherical shapes when $X>0.5$. OVA-LYZ co-aggregates was observed most characteristically in $X=0.5$. In summary, OVA alone and LYZ alone are prone to form small aggregates due to itself electrostatic repulsion resulting in soluble state, while OVA and 
LYZ mixtures form large aggregates with a hierarchical network between small aggregates. As described above, the large aggregates were attributed to the affinity of LYZ with unfolded OVA and intermolecular disulfide bonds between LYZ and OVA molecules.

\section{Discussion}

\subsection{Driving forces for co-aggregation}

First, we discuss the simplest case of aggregates between native OVA (nOVA) and native LYZ (nLYZ). Negatively charged OVA and positively charged LYZ at physiological pH are known to spontaneously associate with each other via electrostatic interactions at extremely low ionic strength even at room temperature (Yu et al., 2006). Such aggregates, called coacervates, can be reversibly dissolved at increasing ionic concentration above $50 \mathrm{mM}$ (Croguennec, Tavares, \& Bouhallab, 2016; Salvatore, Croguennec, Bouhallab, Forge, \& Nicolai, 2011). Thus, under our experimental conditions, nOVA and nLYZ did not form precipitates only by mixing because the solutions contained $50 \mathrm{mM}$ Na-phosphate as a buffer.

On the other hand, aggregates were observed when nOVA and nLYZ were mixed and heated at $70^{\circ} \mathrm{C}$. At this temperature, both OVA and LYZ were slightly unfolded (Supplementary Information, Fig. S2). Note that the mixing of OVA and LYZ does not influence their unfolding temperature (Arntfield \& Bernatsky, 1993). In saline at $70^{\circ} \mathrm{C}$, aggregates were formed through hydrophobic interactions rather than through only electrostatic interactions, followed by crosslinking between OVA and LYZ molecules by disulfide bond exchange. Co-aggregation of OVA and LYZ was sensitive to thermal unfolding compared with single protein system of OVA or LYZ. Weak hydrophobic interaction induced by partial unfolding may be amplified by electrostatic attraction between OVA and LYZ. Therefore, the $\mathrm{pH}$ is an important property for co-aggregation such as oppositely charged OVA and LYZ system. In addition, our data indicated that the aggregation of OVA is not affected by the presence of LYZ. Thus, OVA plays a role as a 
trigger for the start of co-aggregation by unfolding itself.

\subsection{Binding region of unfolded OVA and native $L Y Z$}

Heated OVA (hOVA) showed affinity with native LYZ (nLYZ) via non-covalent interactions. The complex of hOVA with nLYZ formed insoluble precipitates. These results indicated that an internal hydrophobic region of OVA exposed by unfolding had affinity for the surface of nLYZ. In fact, the intramolecular hydrophobic region of OVA was identified as ILELPFASGT MSMLVLLPDE VSGLEQLESIINFEK (residues 229-263), named S-peptide, which is capable of binding strongly to the LYZ molecule (Sugimoto et al., 2011). Sugimoto et al. concluded that S-peptide enhances the formation of LYZ aggregation. It should be noted that S-peptide has no cysteine residues, suggesting that non-covalent interactions between unfolded OVA and native LYZ trigger aggregate formation.

In addition, LYZ has a binding site with OVA (Sugimoto et al., 2011). The amino acid sequences of the peptides were identified as RNRCKGTDVQAW (residues 112-123), named M-peptide, which is located in the LYZ surface, and GILQINSRW (residues 54-62), named K-peptide, which is located in the inside near the active site of LYZ. Considering the association between OVA and LYZ at room temperature, S-peptide of the unfolded OVA was exposed on the protein surface, leading to binding to M-peptide of native LYZ.

\subsection{Residual enzyme reaction rate of $L Y Z$}

The enzyme-reaction rate of LYZ of "hOVA + nLYZ" remained at about 90\% (Fig. 6). This raised the following possibilities: (i) the interaction occurred between a non-active site of LYZ and hOVA, and (ii) LYZ sufficiently dissolved from aggregates due to dilution for the enzyme assay. The two cases are discussed below.

(i) The LYZ molecule mostly adopts the native conformation even at $70^{\circ} \mathrm{C}$ (Supplementary Information, Fig. S2). In addition, the active site residues of LYZ are negatively 
charged Glu35 and Asp52. Therefore, K-peptide of LYZ, which is located in the inside of the molecule near the active site, cannot approach the OVA surface. In fact, the binding of M-peptide to OVA is more favorable than K-peptide to OVA for the detection of OVA (Sugawara, Kadoya, Kuramitz, \& Tanaka, 2014). Furthermore, K-peptide of LYZ is known to be a core region of amyloid fibrils (Tokunaga, Sakakibara, Kamada, Watanabe, \& Sugimoto, 2013); this peptide is prone to form aggregates itself rather than to bind to OVA due to its high hydrophobicity (Sugimoto et al., 2011). Thus, LYZ is thought to bind to OVA via the M-peptide.

(ii) The chromatogram of "hOVA + nLYZ" solution shown in Fig. 3 indicated that all of the soluble OVA aggregates were associated with LYZ, leading to formation of precipitates. If Case (ii) is correct, the precipitates dissociate immediately at the time of dilution with substrate solution during the enzyme assay. We investigated the particle size using a laser diffraction particle sizer, showing that the diameter of the "hOVA + nLYZ" sample was actually decreased by $>10$-fold dilution (Supplementary Information, Fig. S6). The size distribution in 100-fold diluted "hOVA + nLYZ" solution was the same as that of "hOVA" solution. Therefore, it was concluded that the precipitates between hOVA and nLYZ are dissociated by dilution, resulting in reactivation of LYZ (Fig. 6). That is, the precipitates were in an unstable colloidal state.

\subsection{Intermolecular disulfide bond exchange during co-heating}

As shown in SDS-PAGE analyses (Fig. 5), the disulfide bonds were formed between OVA and LYZ molecules during heat treatment. The OVA molecule has four sulfhydryl groups and one disulfide bond buried in the intramolecular core. LYZ has four disulfide bonds in the inside. A previous study indicated that heat treatment at $70^{\circ} \mathrm{C}$ exposed free sulfhydryl groups of OVA to the solvent resulting in induction of a sulfhydryl-disulfide exchange reaction between OVA and LYZ, which caused irreversible aggregate formation (Matsudomi et al., 1986). As shown in Fig. 7, the abundant LYZ in co-aggregates may be attributed to the exchange reaction between a sulfhydryl group of OVA and a disulfide bond of LYZ. The many disulfide bonds in 
LYZ play a key role in connection of soluble OVA aggregates. Sulfhydryl-disulfide exchange reaction between OVA and LYZ propagates new sulfhydryl group of LYZ, leading to formation of large aggregates with crosslinked network.

\subsection{Process of co-aggregation}

Figure 9 shows the molecular mechanism of co-aggregation between OVA and LYZ. The process of co-aggregation includes the following steps: (i) the unfolding of OVA, (ii) the association of unfolded OVA and LYZ via non-covalent interaction, (iii) the insolubilization of the complexes due to the colloidal instability, (iv) the additional binding of LYZ induced by exchange reaction between sulfhydryl groups of unfolded OVA and disulfide bonds of LYZ, and (v) the growth of aggregates due to the formation of intermolecular disulfide bonds across the OVA-LYZ colloids. Sulfhydryl-disulfide exchange reaction between OVA and LYZ in the step (iv) generated new sulfhydryl groups of LYZ. Sulfhydryl groups were propagated by the exchange reaction and contributed to the growth of the aggregate by the formation of intermolecular disulfide bonds in the step (v). The hierarchical aggregation was important for understanding of co-aggregation by OVA and LYZ. For example, if the solution contained LYZ or OVA alone, small aggregates were observed. These small aggregates cannot grow due to the balance between short-range attraction and long-range electrostatic repulsion (A. I. Campbell, Anderson, van Duijneveldt, \& Bartlett, 2005; Mossa, Sciortino, Tartaglia, \& Zaccarelli, 2004). It is important to note that the thermal unfolding of OVA itself does not influence the mixture with LYZ, while that of LYZ markedly facilitates mixture with OVA. This is because aggregation occurs by both sulfhydryl-disulfide exchange reaction and affinity via non-covalent interactions with unfolded OVA.

\section{Conclusions}


The thermal aggregation rate of OVA was not dependent on co-existing LYZ concentration. In contrast, the thermal aggregation rate of LYZ was enhanced by increases in the co-existing OVA concentration. Native LYZ formed precipitates with unfolded OVA by non-covalent interactions even at room temperature. The precipitates were labile and unstable, and could be dissociated by 10 -fold dilution. In addition to the affinity of unfolded OVA for native LYZ, intermolecular disulfide bonds were formed between OVA and LYZ during co-heating. These results indicated that the unfolding of OVA triggered the collaborative aggregation of OVA and LYZ via the affinity of unfolded OVA for LYZ and sulfhydryl-disulfide exchange reaction between OVA and LYZ. The unfolded OVA plays a crucial role in the nucleus of LYZ aggregation before the growth of large aggregates. The precise mechanism of co-aggregation provides information on control of aggregation and gelation of egg white proteins. It will be future issues to clarify relations of this co-aggregation phenomenon and gel properties of egg white protein. Finally, we would like to emphasize that the molecular mechanism of co-aggregation between heterogeneous proteins cannot be extrapolated by investigation of the aggregation of a single protein. We will further focus other combinations of egg white proteins to understand the gelation and aggregation of complex protein systems.

\section{Acknowledgments}

The authors are grateful to the University of Tsukuba and Kewpie Corporation for financial support.

\section{References}

Arntfield, S. D., \& Bernatsky, A. (1993). Characteristics of heat-induced networks for mixtures of ovalbumin and lysozyme. Journal of Agricultural and Food Chemistry, 41(12), $2291-2295$.

Bouhallab, S., \& Croguennec, T. (2014). Spontaneous assembly and induced aggregation of 
food proteins. Advances in Polymer Science, 256, 67-102.

Campanella, B., Onor, M., D’Ulivo, A., Giannarelli, S., \& Bramanti, E. (2014). Impact of protein concentration on the determination of thiolic groups of ovalbumin: A size exclusion chromatography-chemical vapor generation-atomic fluorescence spectrometry study via mercury labeling. Analytical Chemistry, 86(4), 2251-2256.

Campbell, A. I., Anderson, V. J., van Duijneveldt, J. S., \& Bartlett, P. (2005). Dynamical arrest in attractive colloids: The effect of long-range repulsion. Physical Review Letters, 94(20).

Campbell, L., Raikos, V., \& Euston, S. R. (2003). Modification of functional properties of egg-white proteins. Food / Nahrung, 47(6), 369-376.

Croguennec, T., Renault, A., Beaufils, S., Dubois, J.-J., \& Pezennec, S. (2007). Interfacial properties of heat-treated ovalbumin. Journal of Colloid and Interface Science, 315(2), $627-636$.

Croguennec, T., Tavares, G. M., \& Bouhallab, S. (2016). Heteroprotein complex coacervation: A generic process. Advances in Colloid and Interface Science. Retrieved from http://www.sciencedirect.com/science/article/pii/S0001868616301452

De Bernardez Clark, E., Hevehan, D., Szela, S., \& Maachupalli-Reddy, J. (1998). Oxidative renaturation of hen egg-white lysozyme. folding vs aggregation. Biotechnology Progress, 14(1), 47-54.

Guérin-Dubiard, C., Pasco, M., Mollé, D., Désert, C., Croguennec, T., \& Nau, F. (2006). Proteomic analysis of hen egg white. Journal of Agricultural and Food Chemistry, 54(11), $3901-3910$.

Handa, A., Hayashi, K., Shidara, H., \& Kuroda, N. (2001). Correlation of the protein structure and gelling properties in dried egg white products. Journal of Agricultural and Food Chemistry, 49(8), 3957-3964.

Handa, A., Takahashi, K., Kuroda, N., \& Froning, G. W. (1998). Heat-induced egg white gels as affected by pH. Journal of Food Science, 63(3), 403-407. 
Hirano, A., Hamada, H., Okubo, T., Noguchi, T., Higashibata, H., \& Shiraki, K. (2007). Correlation between thermal aggregation and stability of lysozyme with salts described by molar surface tension increment: an exceptional propensity of ammonium salts as aggregation suppressor. The Protein Journal, 26(6), 423-433.

Huntington, J. A., \& Stein, P. E. (2001). Structure and properties of ovalbumin. Journal of Chromatography B: Biomedical Sciences and Applications, 756(1-2), 189-198.

Iwashita, K., Inoue, N., Handa, A., \& Shiraki, K. (2015). Thermal aggregation of hen egg white proteins in the presence of salts. The Protein Journal, 34(3), 212-219.

Kawachi, Y., Kameyama, R., Handa, A., Takahashi, N., \& Tanaka, N. (2013). Role of the $\mathrm{N}$-terminal amphiphilic region of ovalbumin during heat-induced aggregation and gelation. Journal of Agricultural and Food Chemistry, 61(36), 8668-8675.

Kudou, M., Shiraki, K., Fujiwara, S., Imanaka, T., \& Takagi, M. (2003). Prevention of thermal inactivation and aggregation of lysozyme by polyamines. European Journal of Biochemistry / FEBS, 270(22), 4547-4554.

Le Floch-Fouéré, C., Pezennec, S., Lechevalier, V., Beaufils, S., Desbat, B., Pézolet, M., \& Renault, A. (2009). Synergy between ovalbumin and lysozyme leads to non-additive interfacial and foaming properties of mixtures. Food Hydrocolloids, 23(2), 352-365.

Lechevalier, V., Croguennec, T., Pezennec, S., Guérin-Dubiard, C., Pasco, M., \& Nau, F. (2003). Ovalbumin, ovotransferrin, lysozyme: three model proteins for structural modifications at the air-water interface. Journal of Agricultural and Food Chemistry, 51(21), 6354-6361.

Mann, K. (2007). The chicken egg white proteome. PROTEOMICS, 7(19), 3558-3568.

Matsuda, T., Watanabe, K., \& Sato, Y. (1982). Interaction between ovomucoid and lysozyme. Journal of Food Science, 47(2), 637-641.

Matsudomi, N., Yamamura, Y., \& Kobayashi, K. (1986). Heat-induced aggregation between ovalbumin and lysozyme. Agricultural and Biological Chemistry, 50(6), 1389-1395.

Matsudomi, N., Yamamura, Y., \& Kobayashi, K. (1987). Aggregation between lysozyme and 
heat-denatured ovalbumin. Agricultural and Biological Chemistry, 51(7), 1811-1817.

Matsuoka, T., Hamada, H., Matsumoto, K., \& Shiraki, K. (2009). Indispensable structure of solution additives to prevent inactivation of lysozyme for heating and refolding. Biotechnology Progress, 25(5), 1515-1524.

Matsuoka, T., Tomita, S., Hamada, H., \& Shiraki, K. (2007). Amidated amino acids are prominent additives for preventing heat-induced aggregation of lysozyme. Journal of Bioscience and Bioengineering, 103(5), 440-443.

Mine, Y. (1995). Recent advances in the understanding of egg white protein functionality. Trends in Food Science \& Technology, 6(7), 225-232.

Mine, Y., Noutomi, T., \& Haga, N. (1990). Thermally induced changes in egg white proteins. Journal of Agricultural and Food Chemistry, 38(12), 2122-2125.

Mizutani, K., Chen, Y., Yamashita, H., Hirose, M., \& Aibara, S. (2006). Thermostabilization of ovotransferrin by anions for pasteurization of liquid egg white. Bioscience, Biotechnology, and Biochemistry, 70(8), 1839-1845.

Mossa, S., Sciortino, F., Tartaglia, P., \& Zaccarelli, E. (2004). Ground-state clusters for short-range attractive and long-range repulsive potentials. Langmuir, 20(24), 10756-10763.

Nieuwland, M., Bouwman, W. G., Pouvreau, L., Martin, A. H., \& de Jongh, H. H. J. (2016). Relating water holding of ovalbumin gels to aggregate structure. Food Hydrocolloids, 52, 87-94. http://doi.org/10.1016/j.foodhyd.2015.06.018

Nigen, M., Croguennec, T., \& Bouhallab, S. (2009). Formation and stability of $\alpha$-lactalbumin-lysozyme spherical particles: Involvement of electrostatic forces. Food Hydrocolloids, 23(2), 510-518.

Offengenden, M., \& Wu, J. (2013). Egg white ovomucin gels: structured fluids with weak polyelectrolyte properties. $R S C A d v ., 3(3), 910-917$.

Okanojo, M., Shiraki, K., Kudou, M., Nishikori, S., \& Takagi, M. (2005). Diamines prevent 
thermal aggregation and inactivation of lysozyme. Journal of Bioscience and Bioengineering, 100(5), 556-561.

Salvatore, D., Croguennec, T., Bouhallab, S., Forge, V., \& Nicolai, T. (2011). Kinetics and structure during self-assembly of oppositely charged proteins in aqueous solution. Biomacromolecules, 12(5), 1920-1926.

Shiraki, K., Kudou, M., Fujiwara, S., Imanaka, T., \& Takagi, M. (2002). Biophysical Effect of Amino Acids on the Prevention of Protein Aggregation. Journal of Biochemistry, 132(4), $591-595$.

Shiraki, K., Kudou, M., Nishikori, S., Kitagawa, H., Imanaka, T., \& Takagi, M. (2004). Arginine ethylester prevents thermal inactivation and aggregation of lysozyme. European Journal of Biochemistry / FEBS, 271(15), 3242-3247.

Shiraki, K., Kudou, M., Sakamoto, R., Yanagihara, I., \& Takagi, M. (2005). Amino Acid esters prevent thermal inactivation and aggregation of lysozyme. Biotechnology Progress, 21(2), $640-643$.

Stevens, L. (1991). Egg white proteins. Comparative Biochemistry and Physiology Part B: Comparative Biochemistry, 100(1), 1-9.

Sugawara, K., Kadoya, T., Kuramitz, H., \& Tanaka, S. (2014). Voltammetric detection of ovalbumin using a peptide labeled with an electroactive compound. Analytica Chimica Acta, 834, 37-44.

Sugimoto, Y., Kamada, Y., Tokunaga, Y., Shinohara, H., Matsumoto, M., Kusakabe, T., Ohkuri T., \& Ueda, T. (2011). Aggregates with lysozyme and ovalbumin show features of amyloid-like fibrils. Biochemistry and Cell Biology, 89(6), 533-544.

Sun, Y., \& Hayakawa, S. (2002). Heat-induced gels of egg white/ovalbumins from five avian species: thermal aggregation, molecular forces involved, and rheological properties. Journal of Agricultural and Food Chemistry, 50(6), 1636-1642.

Tani, F., Shirai, N., Venelle, F., Yasumoto, K., Onishi, T., \& Doi, E. (1997). Temperature control 
for kinetic refolding of heat-denatured ovalbumin. Protein Science, 6(7), 1491-1502.

Tokunaga, Y., Sakakibara, Y., Kamada, Y., Watanabe, K., \& Sugimoto, Y. (2013). Analysis of core region from egg white lysozyme forming amyloid fibrils. International Journal of Biological Sciences, 9(2), 219-227.

Tomczyńska-Mleko, M., Nishinari, K., \& Handa, A. (2014). $\mathrm{Ca}^{2+}$-induced egg white isolate gels with various microstructure. Food Science and Technology Research, 20(6), 1207-1212.

Urbonaite, V., de Jongh, H. H., van der Linden, E., \& Pouvreau, L. (2015). Protein aggregates may differ in water entrapment but are comparable in water confinement. Journal of Agricultural and Food Chemistry, 63(40), 8912-8920.

Van der Plancken, I., Van Loey, A., \& Hendrickx, M. E. (2005). Changes in sulfhydryl content of egg white proteins due to heat and pressure treatment. Journal of Agricultural and Food Chemistry, 53(14), 5726-5733.

Weijers, M., Barneveld, P. A., Cohen Stuart, M. A., \& Visschers, R. W. (2003). Heat-induced denaturation and aggregation of ovalbumin at neutral $\mathrm{pH}$ described by irreversible first-order kinetics. Protein Science, 12(12), 2693-2703.

Yamashita, H., Ishibashi, J., Hong, Y.-H., \& Hirose, M. (1998). Involvement of ovotransferrin in the thermally induced gelation of egg white at around $65^{\circ} \mathrm{C}$. Bioscience, Biotechnology, and Biochemistry, 62(3), 593-595.

Yu, S., Yao, P., Jiang, M., \& Zhang, G. (2006). Nanogels prepared by self-assembly of oppositely charged globular proteins. Biopolymers, 83(2), 148-158. 

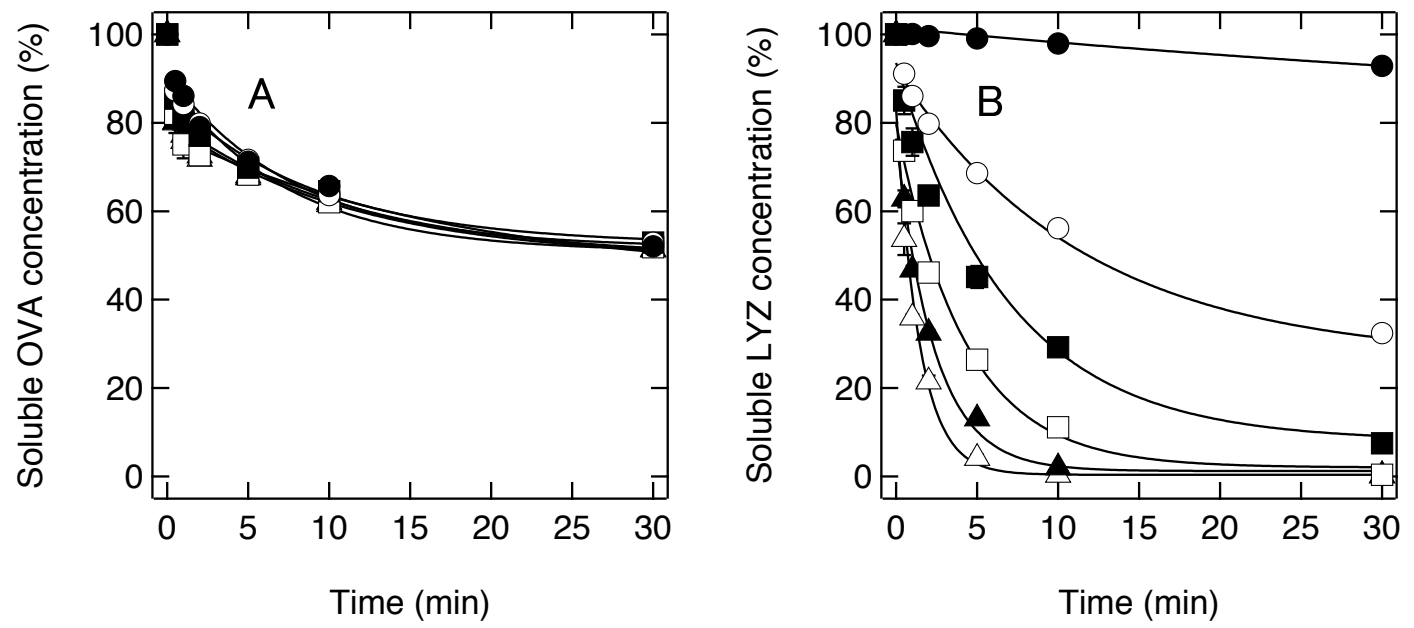

Fig. 1. Soluble protein concentration of OVA-LYZ mixture after heating at $70^{\circ} \mathrm{C}$ for various periods. (A) Samples contained $50 \mu \mathrm{M}$ OVA and 0 (closed circles), 10 (open circles), 20 (closed squares), 30 (open squares), 40 (closed triangles), and 50 (open triangles) $\mu \mathrm{M}$ LYZ. (B) Samples contained $50 \mu \mathrm{M} \mathrm{LYZ} \mathrm{with} 0$ (closed circles), 10 (open circles), 20 (closed squares), 30 (open squares), 40 (closed triangles), and 50 (open triangles) $\mu \mathrm{M}$ OVA.
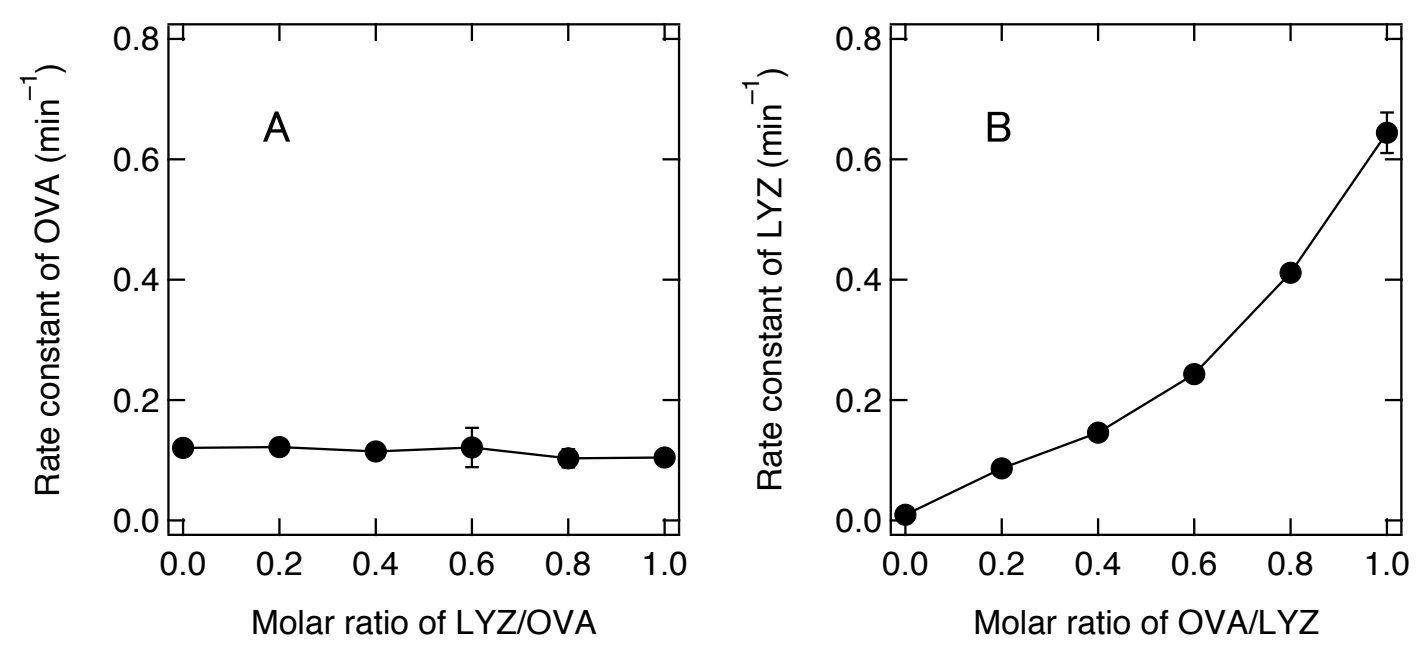

Fig. 2. Aggregation rate constants of OVA and LYZ. (A) The aggregation rate constants of OVA in the presence of various ratios of LYZ. (B) The aggregation rate constants of LYZ in the presence of various ratios of OVA. 


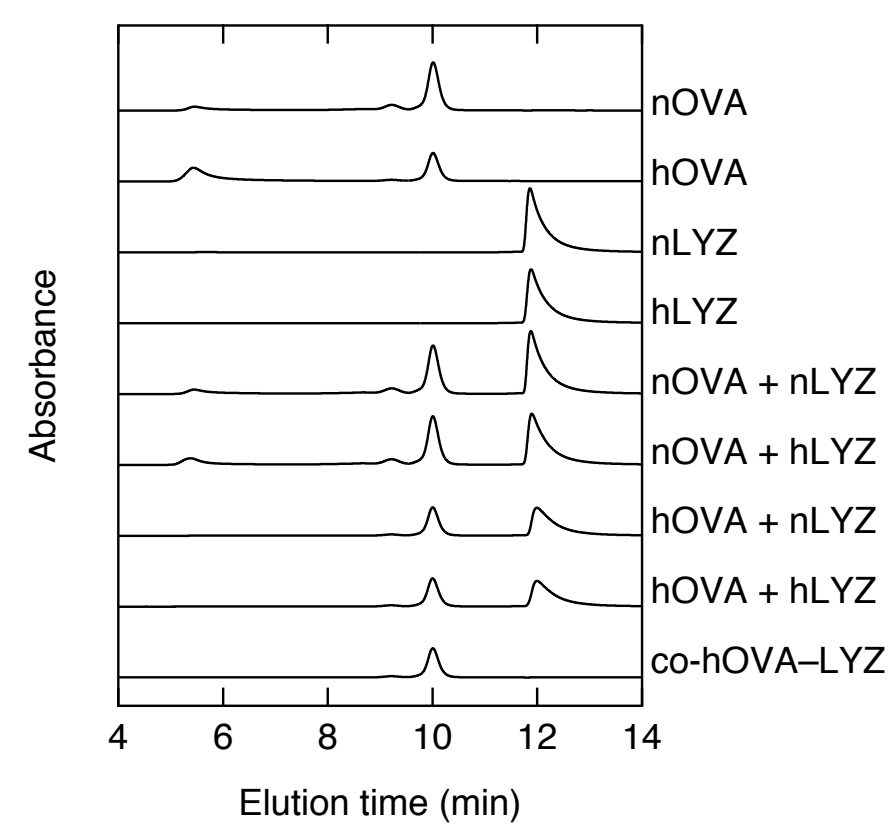

Fig. 3. Size exclusion chromatograms of the supernatant of OVA-LYZ mixture. nOVA, native OVA. hOVA, OVA heated at $70^{\circ} \mathrm{C}$ for $30 \mathrm{~min}$. nLYZ, native LYZ. hLYZ, LYZ heated at $70^{\circ} \mathrm{C}$ for $30 \mathrm{~min}$. The + symbol denotes the mixed sample of the native protein or the heat-treated protein. "co-hOVA-LYZ" indicates the sample in which nOVA and nLYZ mixture was heated at $70^{\circ} \mathrm{C}$ for $30 \mathrm{~min}$.

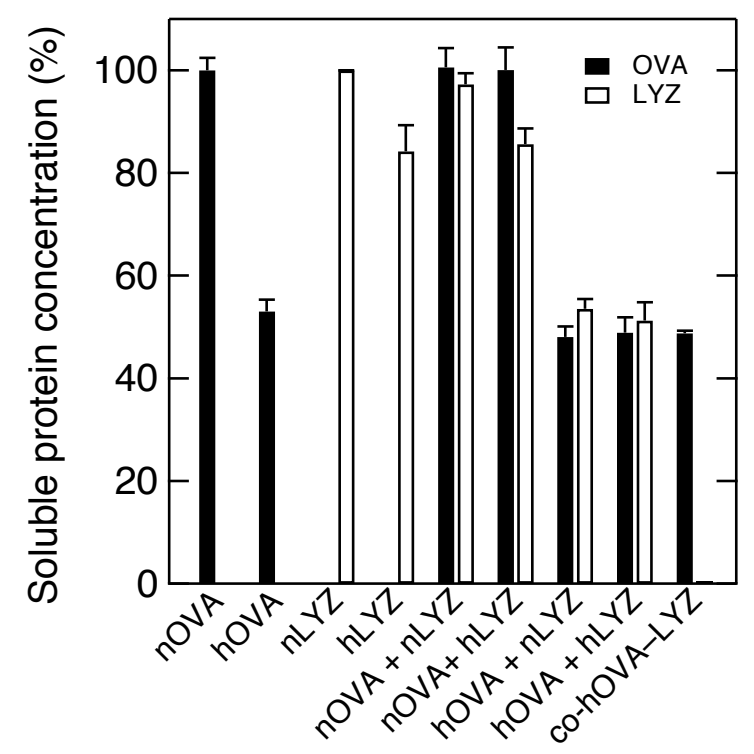

Fig. 4. Soluble protein concentration determined by the chromatograms in Fig. 3. 

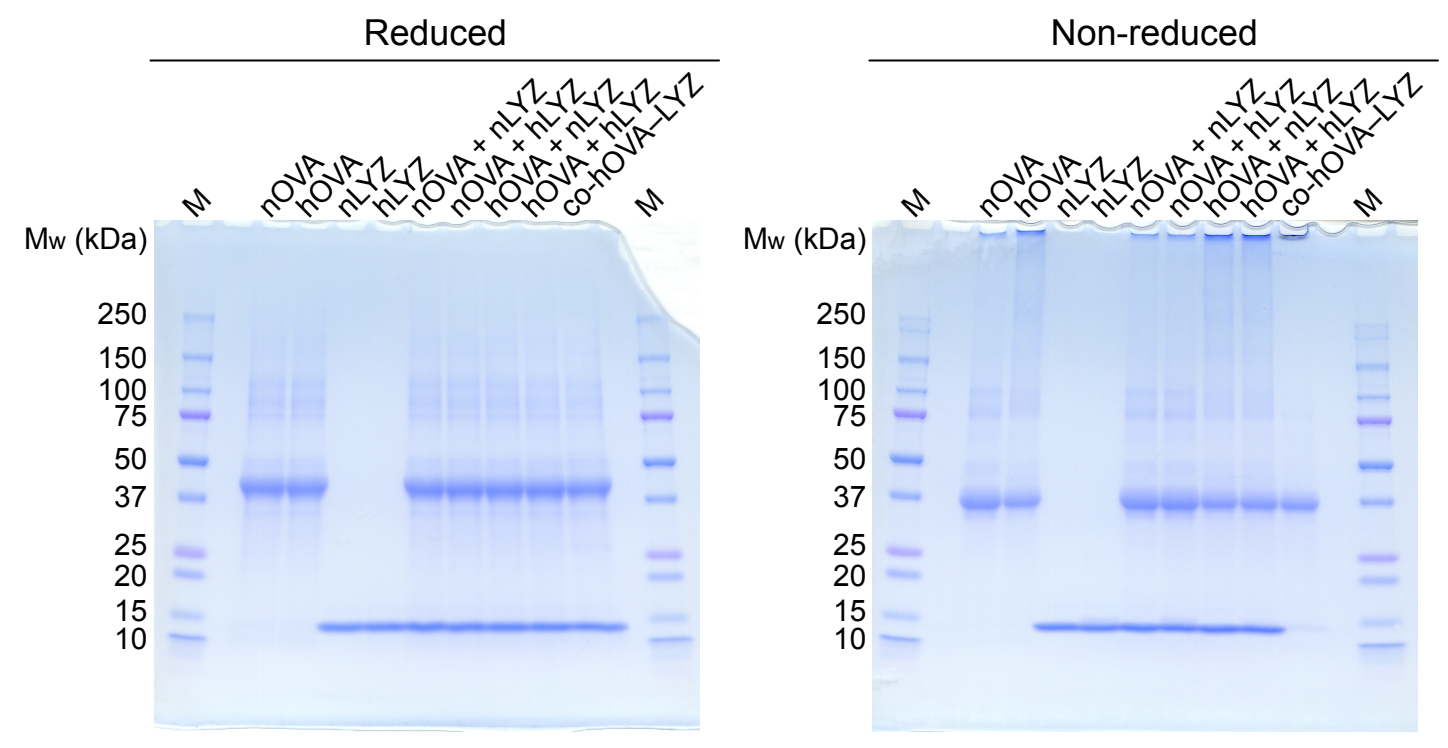

Fig. 5. SDS-PAGE analysis of reduced and non-reduced OVA-LYZ mixtures. nOVA, native OVA. hOVA, OVA heated at $70^{\circ} \mathrm{C}$ for $30 \mathrm{~min}$. nLYZ, native LYZ. hLYZ, LYZ heated at $70^{\circ} \mathrm{C}$ for $30 \mathrm{~min}$. The + symbol denotes the mixed sample of the native protein or the heat-treated protein. "co-hOVA-LYZ" indicates the sample in which nOVA and nLYZ mixture was heated at $70^{\circ} \mathrm{C}$ for $30 \mathrm{~min}$. Lane $\mathrm{M}$ shows standard ladder marker.

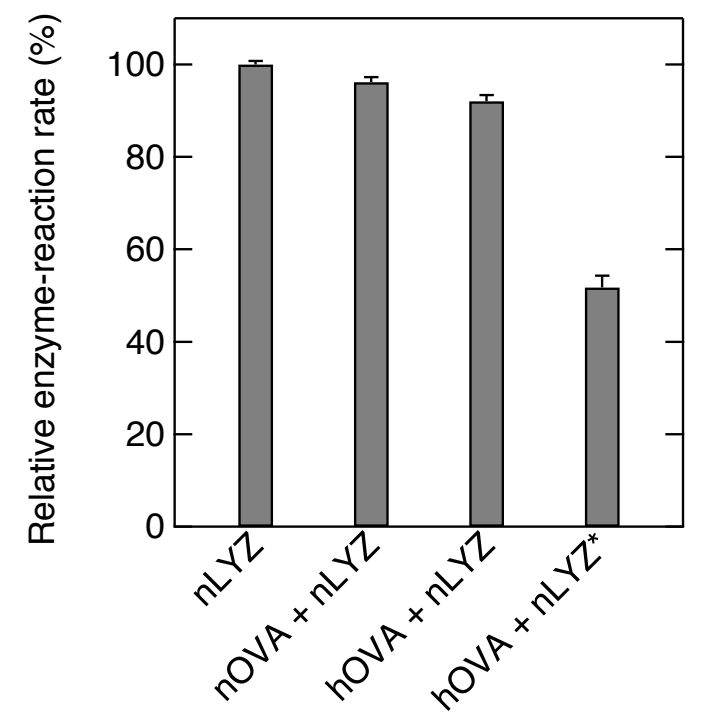

Fig. 6. Enzyme-reaction rate of LYZ with or without OVA. nLYZ, native LYZ. nOVA + nLYZ, native $\mathrm{LYZ}$ with native OVA. hOVA + nLYZ, native LYZ with OVA heated at $70^{\circ} \mathrm{C}$ for $30 \mathrm{~min}$. hOVA + nLYZ*, soluble fraction of "hOVA + nLYZ" obtained by centrifugation at $15,000 \times g$ for $20 \mathrm{~min}$. 


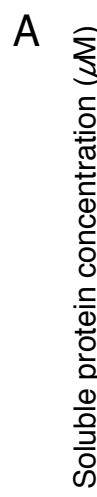

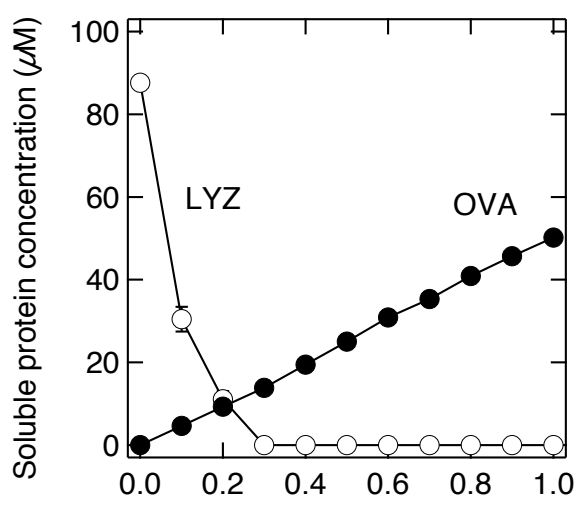

Overall molar fraction of OVA

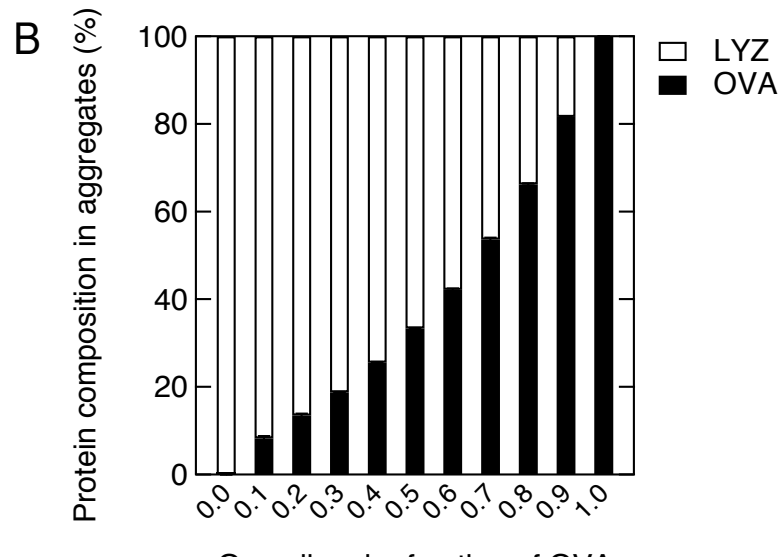

Overall molar fraction of OVA

Fig. 7. (A) Soluble protein concentration of OVA-LYZ mixture after heat treatment. (B) Composition of aggregates in the formed OVA-LYZ mixture. The initial concentration of total protein was $100 \mu \mathrm{M}$. Molar fraction of OVA was [OVA]/([LYZ] $+[\mathrm{OVA}])$.
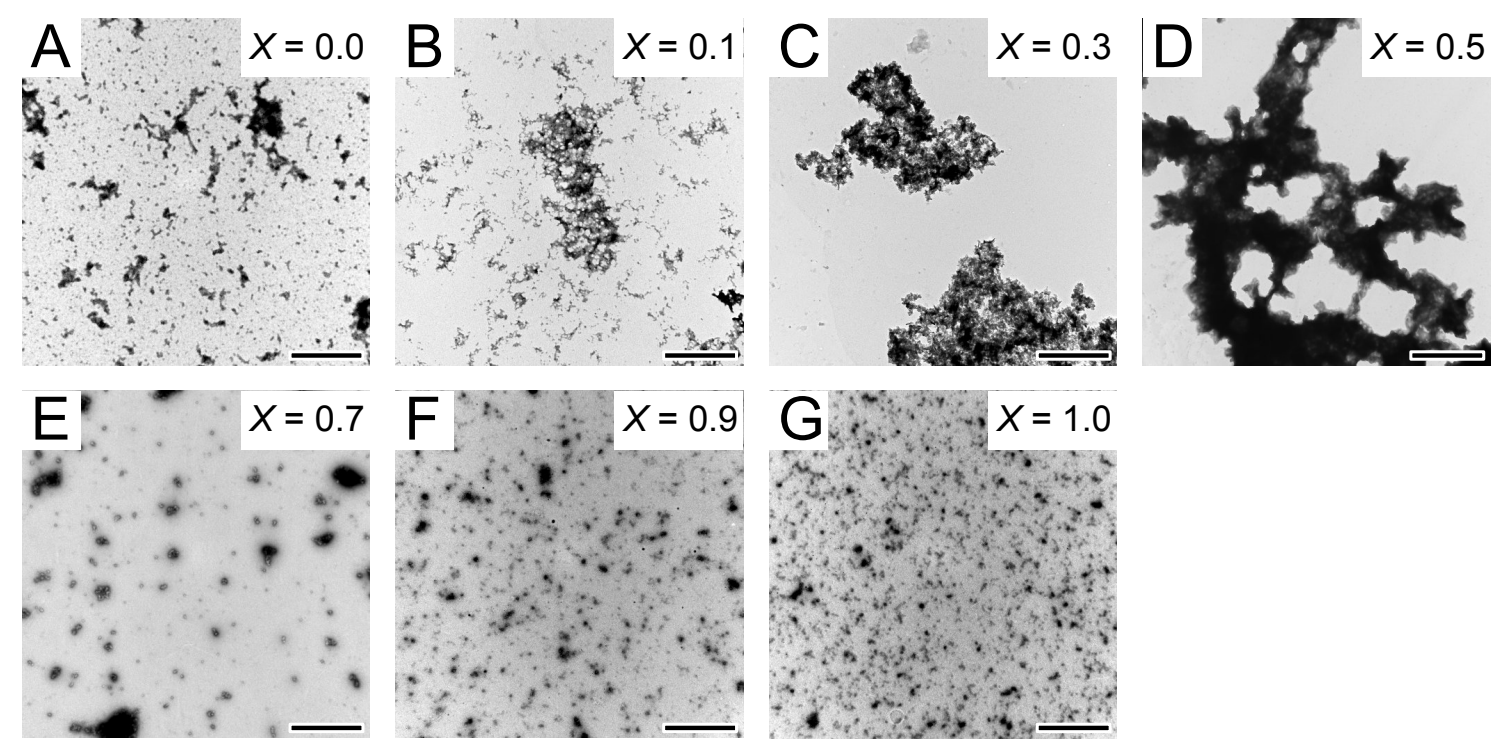

Fig. 8. Morphology of OVA-LYZ mixture aggregation in the molar fraction monitored by TEM. $X=[\mathrm{OVA}] /([\mathrm{LYZ}]+[\mathrm{OVA}])$. The scale bars represent $1 \mu \mathrm{m}$. 
Mixture of OVA and LYZ

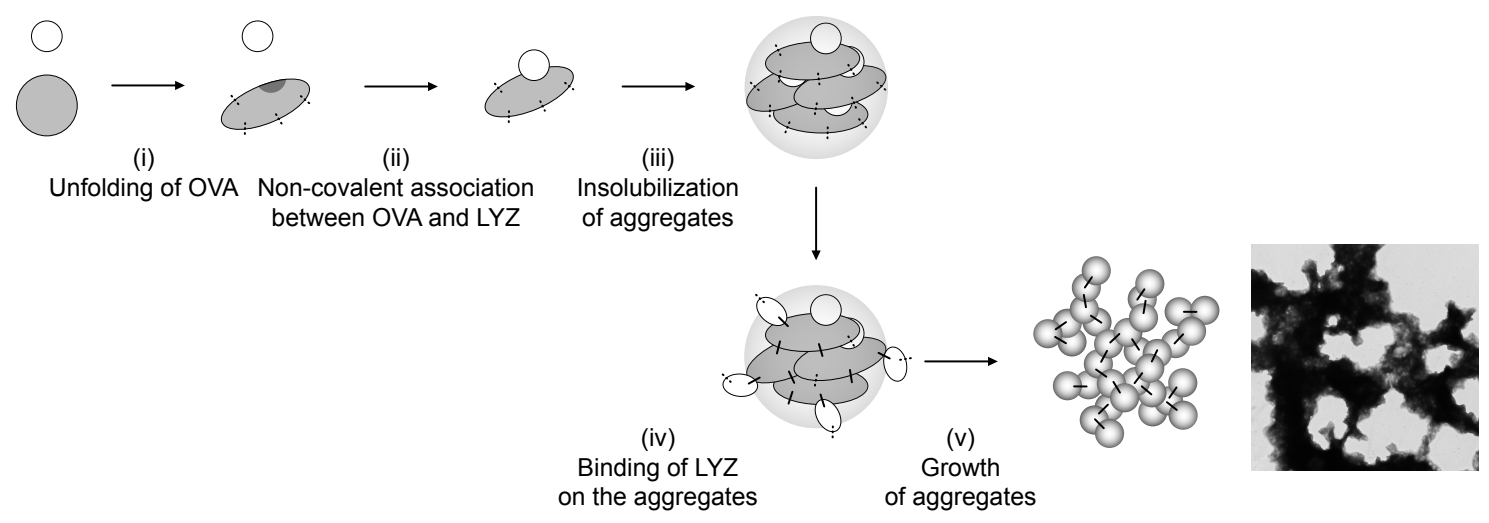

OVA alone

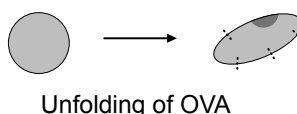

Aggregation

by disulfide crosslinkage
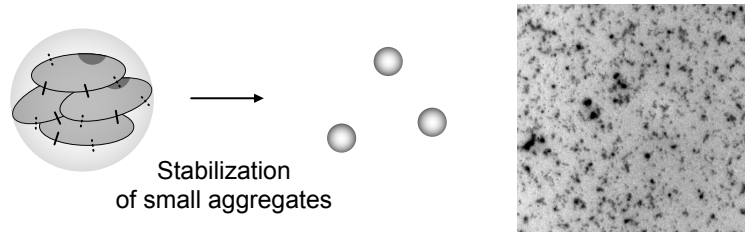

LYZ alone
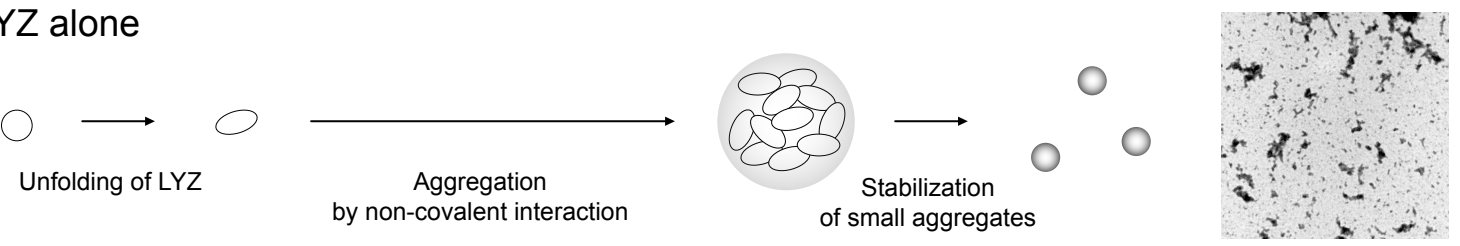

$\bigcirc$ LYZ $\bigcirc$ unfolded LYZ

native OVA

unfolded OVA

binding region for LYZ

.... sulfhydryl group

- disulfide bond

Fig. 9. Schematic diagram of co-aggregation process of OVA and LYZ. 


\section{Supplementary Information}

Sample preparation for thermal aggregation of protein mixtures

A solution of $100 \mu \mathrm{M}$ OVA, LYZ, and bovine serum albumin (BSA) containing $50 \mathrm{mM}$ Na-phosphate buffer (pH 7.0) was heated at $70^{\circ} \mathrm{C}$ for $30 \mathrm{~min}$. The sample solution was diluted 2-fold with buffer solution or mixed with another protein solution at a ratio of 1:1 at room temperature. The sample was immediately centrifuged at $15,000 \times \mathrm{g}$ for $20 \mathrm{~min}$, and then soluble protein concentration in the supernatant was determined by size exclusion chromatography. The sample solution before centrifugation was analyzed by electrophoresis.

\section{Circular dichroism}

Circular dichroism (CD) measurements were performed on a spectropolarimeter (J-720W; Japan Spectroscopic Co. Ltd., Tokyo, Japan) using a Peltier cell holder with a temperature controller (PTC-348W; Japan Spectroscopic Co. Ltd.). A solution of $0.5 \mathrm{mg} \mathrm{mL} \mathrm{m}^{-1} \mathrm{OVA}$, LYZ, and BSA dissolved in $50 \mathrm{mM}$ Na-phosphate buffer ( $\mathrm{pH}$ 7.0) was measured by CD $222 \mathrm{~nm}$ intensity change with an increasing temperature rate of $1.0^{\circ} \mathrm{C} / \mathrm{min}$ using a $1-\mathrm{mm}$ path-length quartz cell.

\section{Distribution of aggregate size}

The size distributions were analyzed using a laser diffraction particle sizer (SALD-2300; Shimadzu, Kyoto, Japan). 


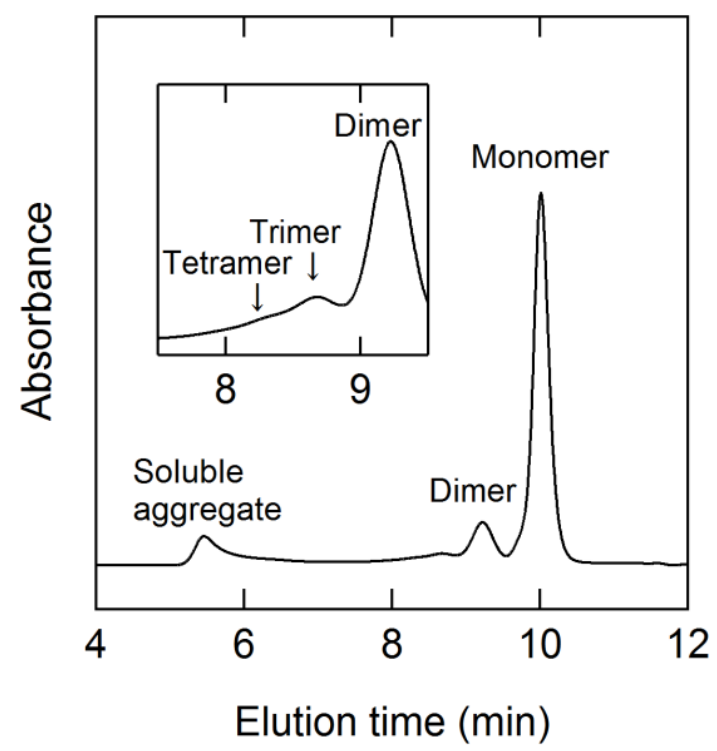

Fig. S1. Size exclusion chromatogram of OVA solution before heat treatment.
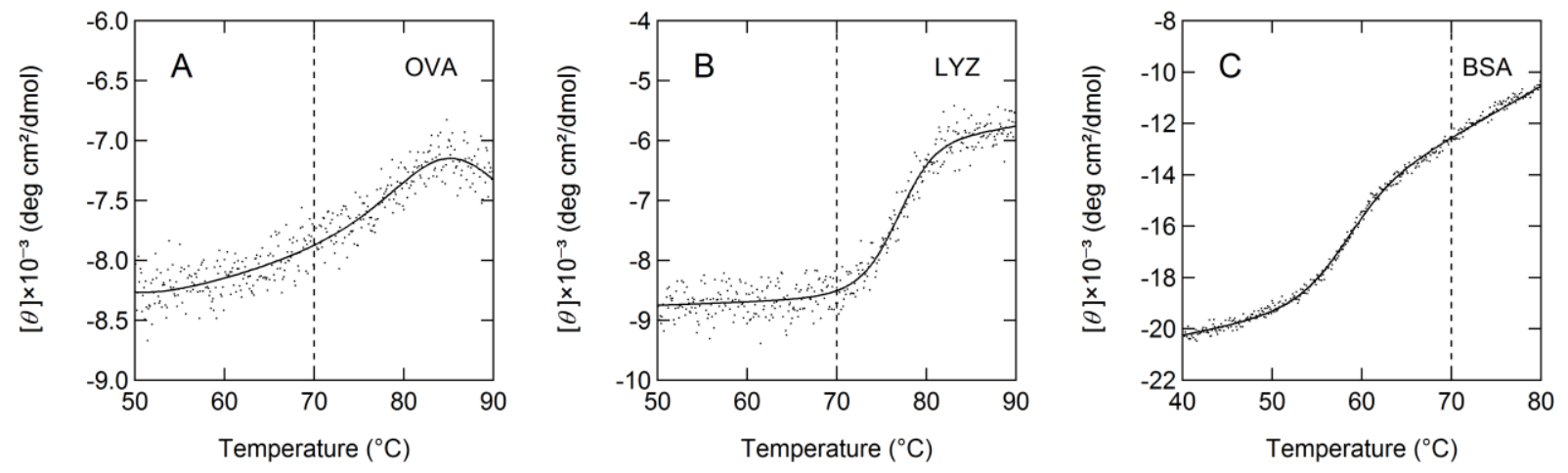

Fig. S2. Thermal unfolding curves of OVA (A), LYZ (B), and BSA (C). 


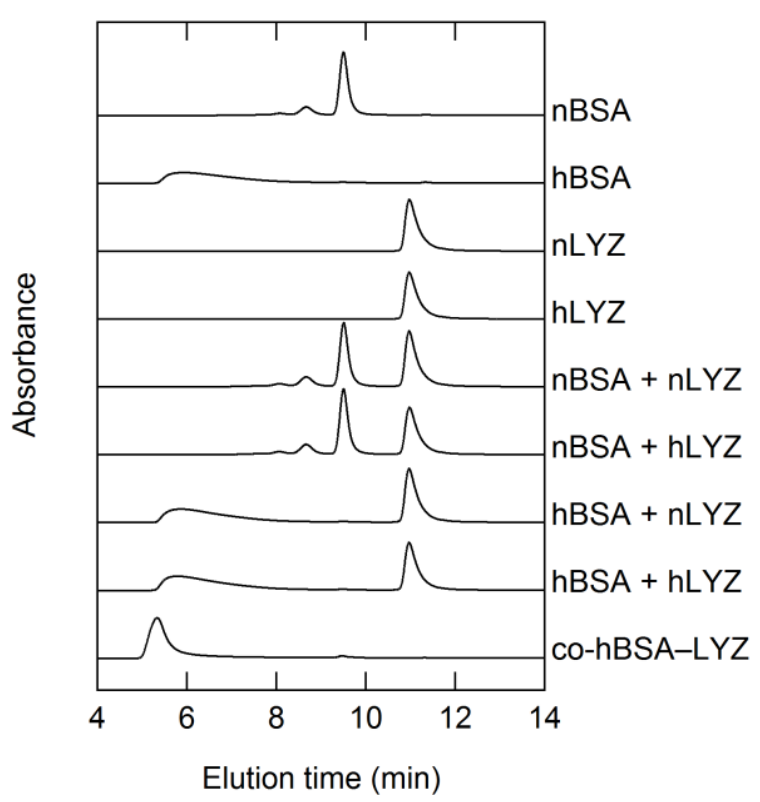

Fig. S3. Size exclusion chromatograms of the supernatant of BSA-LYZ mixture. nBSA, native BSA. hBSA, BSA heated at $70^{\circ} \mathrm{C}$ for $30 \mathrm{~min}$. nLYZ, native LYZ. hLYZ, LYZ heated at $70^{\circ} \mathrm{C}$ for $30 \mathrm{~min}$. The + symbol denotes the mixed sample of the native protein or the heat-treated protein. "co-hBSA-LYZ" indicates the sample in which nBSA and nLYZ mixture was heated at $70^{\circ} \mathrm{C}$ for $30 \mathrm{~min}$.

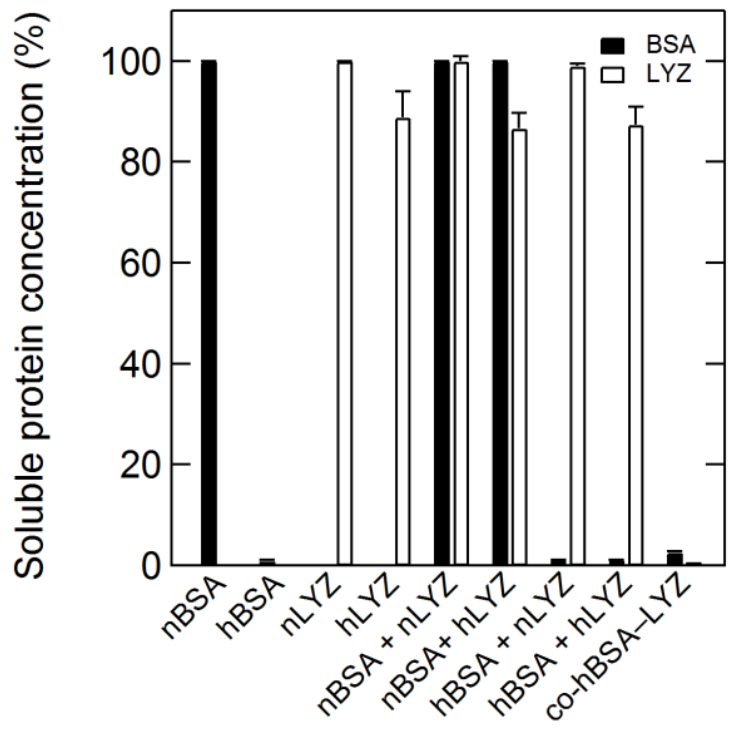

Fig. S4. Soluble protein concentration determined by the chromatograms in Fig. S3. 


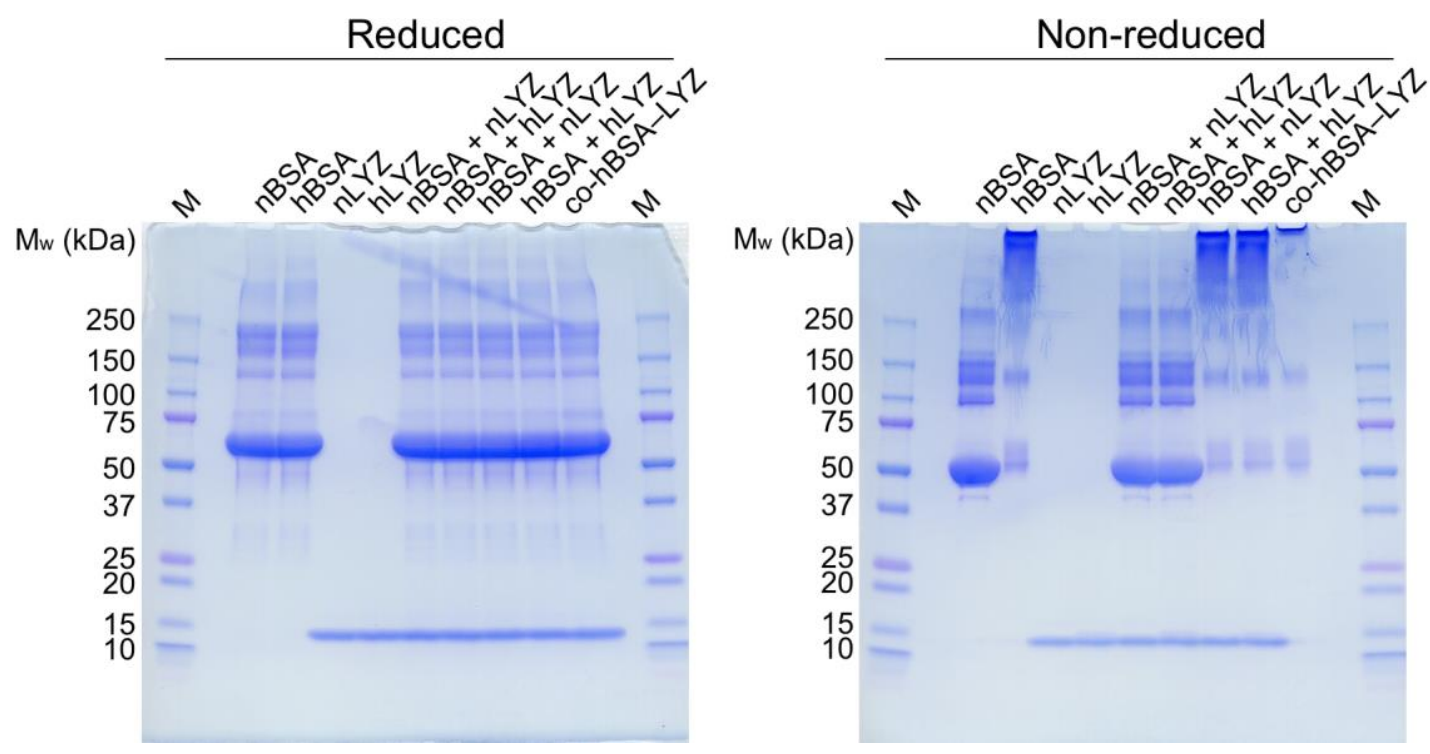

Fig. S5. SDS-PAGE analysis of reduced and non-reduced BSA-LYZ mixtures. nBSA, native BSA. hBSA, BSA heated at $70^{\circ} \mathrm{C}$ for $30 \mathrm{~min}$. nLYZ, native LYZ. hLYZ, LYZ heated at $70^{\circ} \mathrm{C}$ for $30 \mathrm{~min}$. The + symbol denotes the mixed sample of the native protein or the heat-treated protein. "co-hBSA-LYZ" indicates the sample in which nBSA and nLYZ mixture was heated at $70^{\circ} \mathrm{C}$ for $30 \mathrm{~min}$. Lane $\mathrm{M}$ shows standard ladder marker.

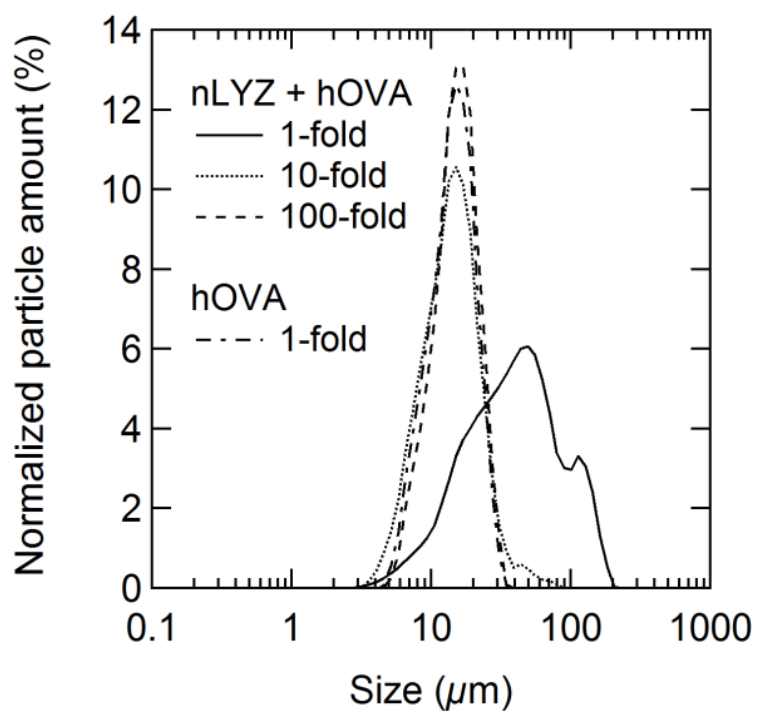

Fig. S6. Size distribution of particles dissolved in the 1-, 10-, and 100-fold diluted "hOVA + nLYZ" and 1-fold "hOVA" solution. 